Supporting Information (SI) for

\title{
Estimation and correction of the blood volume variations of dried blood spots using a postcolumn infused-internal standard strategy with LC-ESI-MS
}

Hsiao-Wei Liao ${ }^{\dagger \dagger}$, Shu-Wen Lin ${ }^{\S, \perp}$, Guan-Yuan Chen ${ }^{\dagger, \ddagger}$, and Ching-Hua Kuo*,†,+,

${ }^{\dagger}$ School of Pharmacy, College of Medicine, National Taiwan University, Taiwan.

*The Metabolomics Core Laboratory, Center of Genomic Medicine, National Taiwan University, Taiwan.

${ }^{\S}$ Graduate Institute of Clinical Pharmacy, College of Medicine, National Taiwan University, Taipei, Taiwan.

${ }^{\perp}$ Department of Pharmacy, National Taiwan University Hospital, Taiwan.

*Corresponding Author

Ching-Hua Kuo

Address: School of Pharmacy, College of Medicine, National Taiwan University, Rm. 418, 4F., No.33, Linsen S. Rd., Chongsheng Dist., Taipei City 100, Taiwan (R.O.C.)

Tel: +886.2 .33668766$

Fax: +886.2 .23919098$

E-mail: kuoch@ntu.edu.tw 
This SI contains the following:

Figure S-1. The minimum response in the first inhibition zone of different blood volume DBS obtained from blood samples (a) without EDTA (b) with EDTA anti-coagulant.

Figure S-2. The structures of 6 tested PCI-ISs and voriconazole.

Figure S-3. The blood volume estimation accuracy of the PCI-IS method.

Figure S-4. The influence of the mass ionization parameters (a) the drying gas temperature, (b) the drying gas flow rate, (c) the nebulizer pressure, and (d) the sheath gas flow rate on the minimum response of the first ion suppression zone of the PCI-IS (posaconazole) chromatogram for 5, 15 and $25 \mu \mathrm{L}$ DBS blood samples.

Figure S-5. The chromatograms of voriconazole at the concentration of LOD and LOQ. 

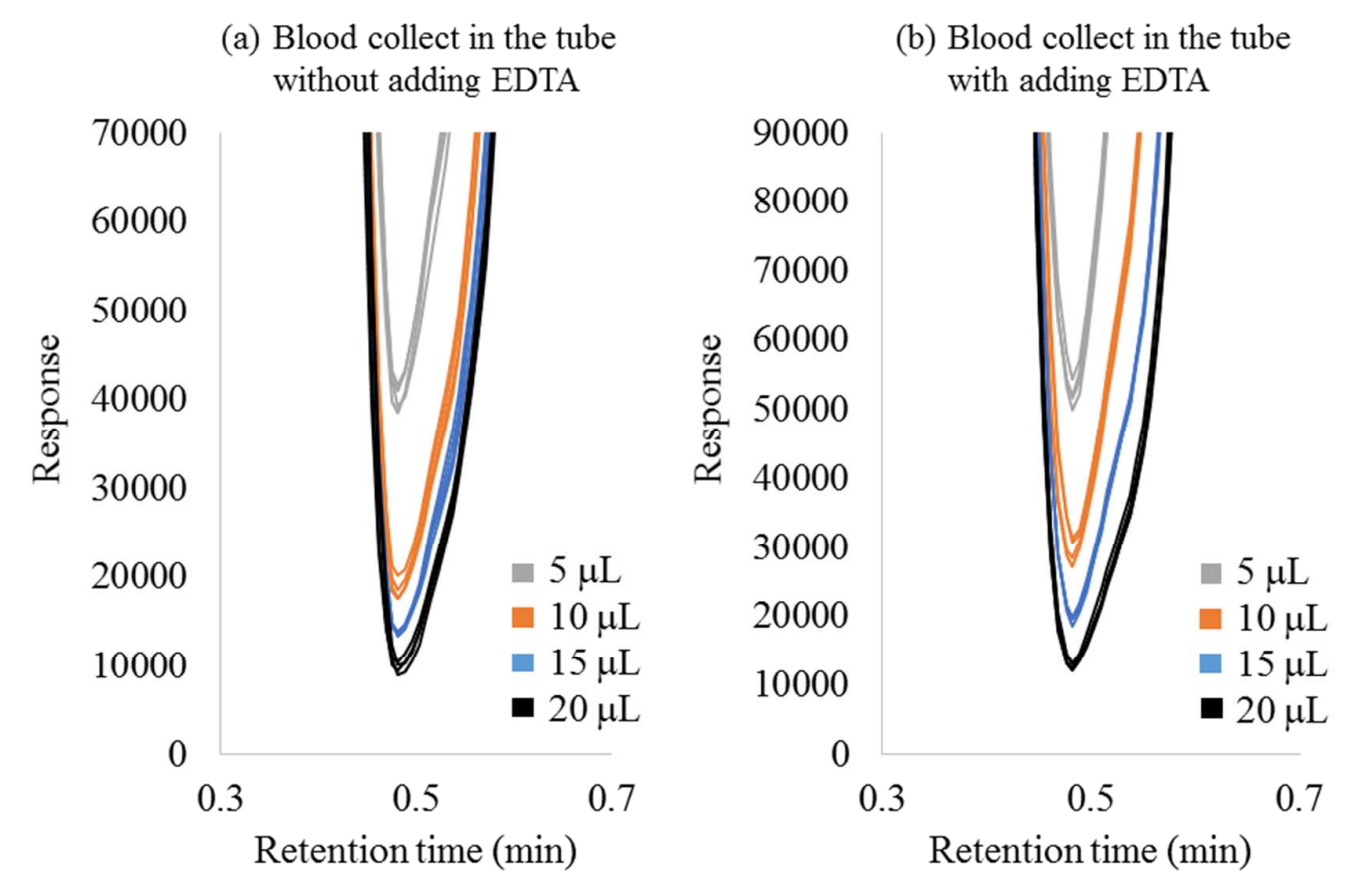

Figure S-1. The minimum response in the first inhibition zone of different blood volume DBS obtained from blood samples (a) without EDTA (b) with EDTA anti-coagulant. 


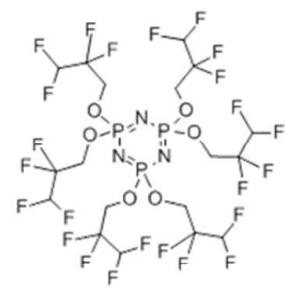

HKP

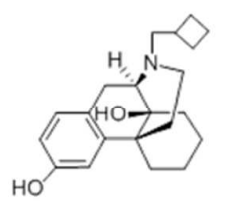

Butorphanol

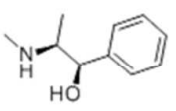

Ephedrine

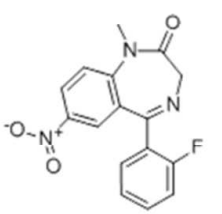

Flunitrazepam

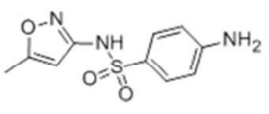

Sulfamethoxazole

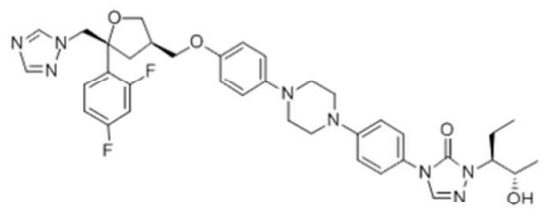

Posaconazole

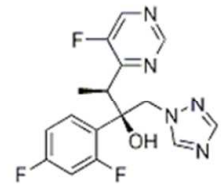

Voriconazole

Figure S-2. The structures of 6 tested PCI-ISs and voriconazole. 


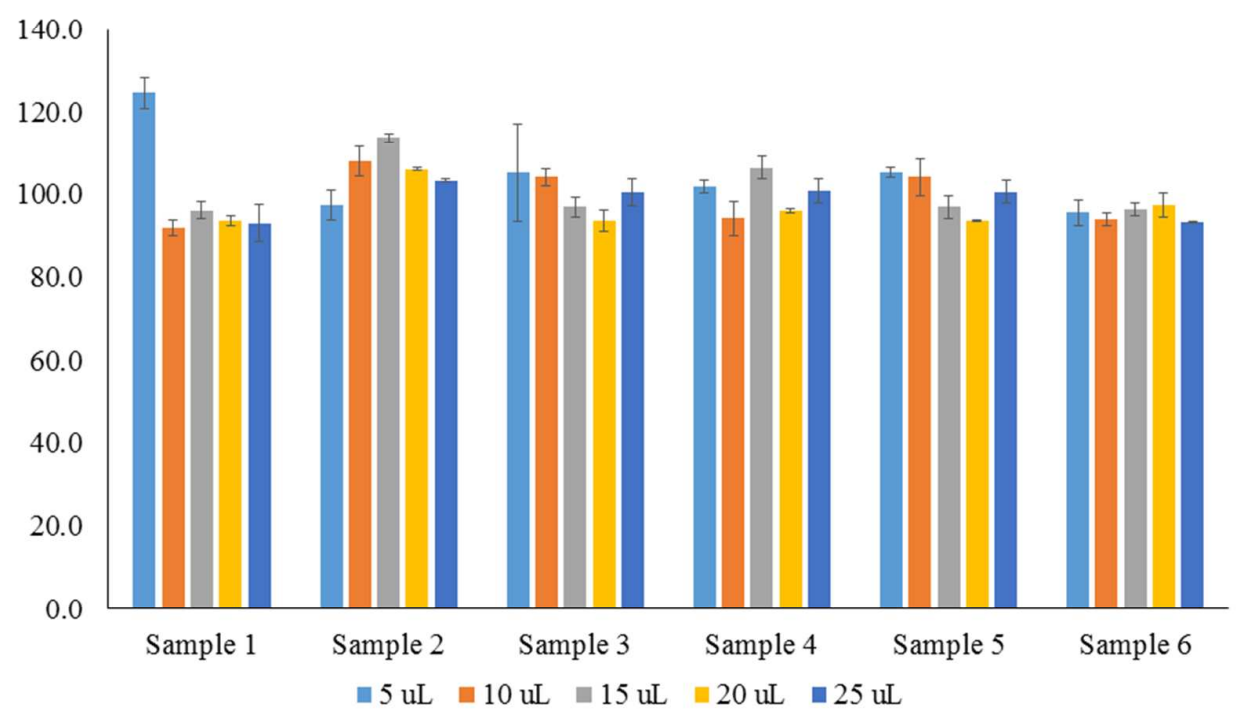

Figure S-3. The blood volume estimation accuracy of the PCI-IS method. 

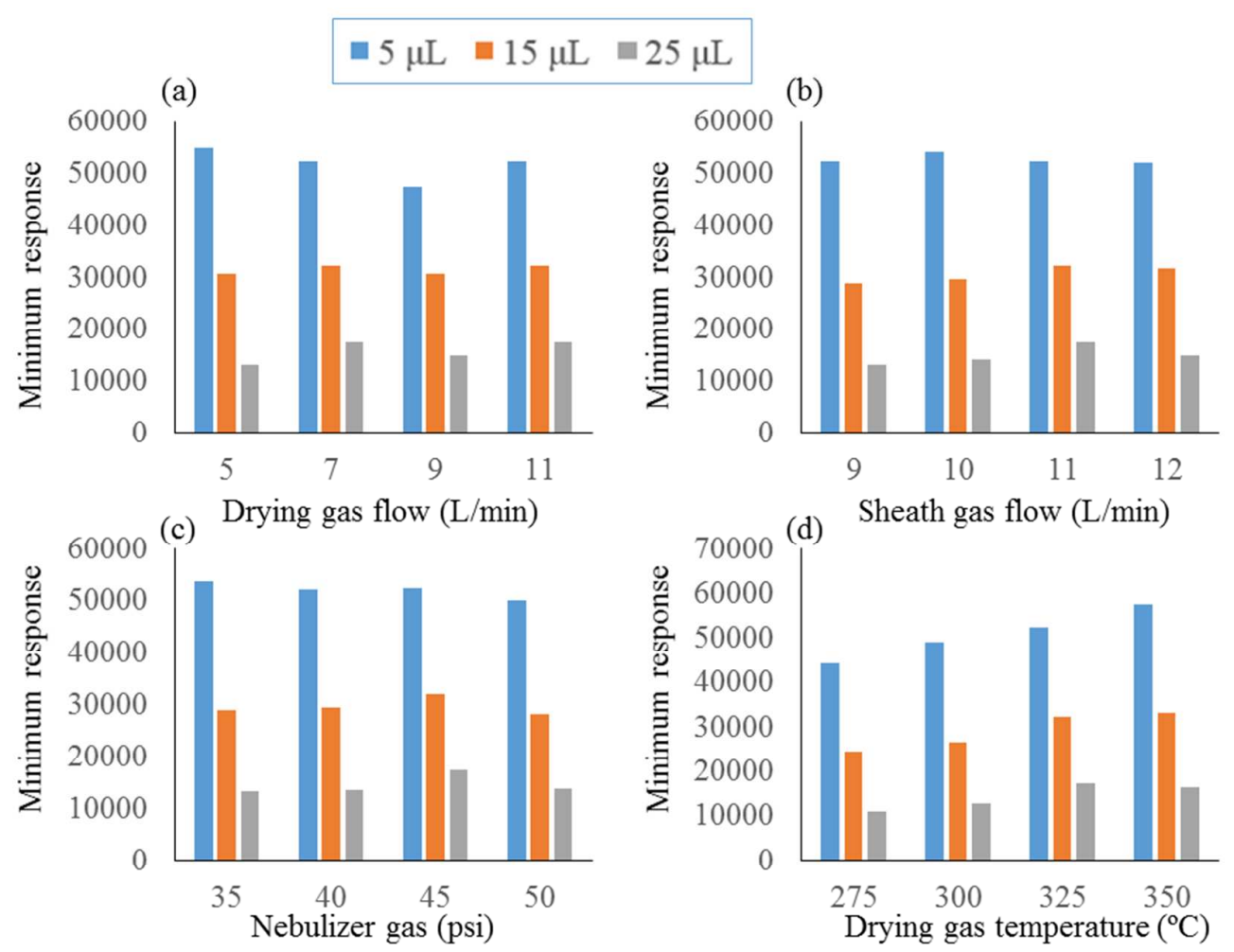

Figure S-4. The influence of the mass ionization parameters (a) the drying gas temperature, (b) the drying gas flow rate, (c) the nebulizer pressure, and (d) the sheath gas flow rate on the minimum response of the first ion suppression zone of the PCI-IS (posaconazole) chromatogram for 5, 15 and $25 \mu \mathrm{L}$ DBS blood samples. 


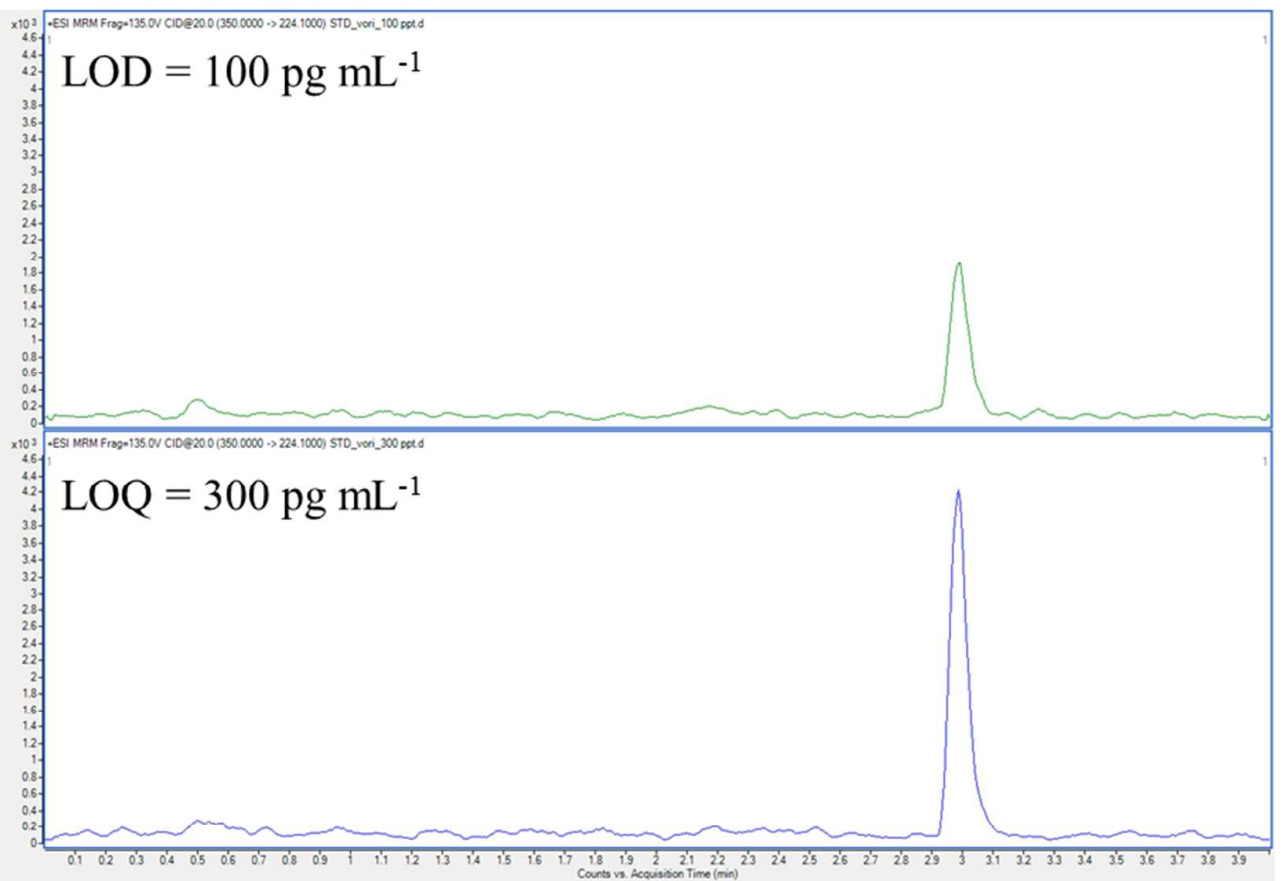

Figure S-5. The chromatograms of voriconazole at the concentration of LOD and LOQ. 
Table S-1. The precision of blood volume estimation of 6 different individuals. ( $n=3$ runs)

\begin{tabular}{lcccccc}
\hline & Sample 1 & Sample 2 & Sample 3 & Sample 4 & Sample 5 & Sample 6 \\
\hline $5 \mathrm{uL}$ & 3.77 & 3.57 & 11.80 & 1.48 & 1.08 & 3.01 \\
$10 \mathrm{uL}$ & 1.86 & 3.67 & 2.04 & 4.11 & 4.50 & 1.60 \\
$15 \mathrm{uL}$ & 1.96 & 1.08 & 2.46 & 2.65 & 2.66 & 1.56 \\
$20 \mathrm{uL}$ & 1.11 & 0.27 & 2.71 & 0.56 & 0.02 & 2.87 \\
$25 \mathrm{uL}$ & 4.38 & 0.24 & 3.13 & 2.89 & 2.74 & 0.20 \\
\hline
\end{tabular}

